\title{
Prevalencia de problemas de sueño en mujeres climatéricas colombianas durante la pandemia COVID-19
}

\section{Prevalence of sleep disorders in menopausal women during the COVID-19 pandemic in Colombia}

Álvaro Monterrosa-Castro, MD, Esp. ${ }^{1}$; Angélica Monterrosa-Blanco, MD $^{2}$

Recibido: 18 de febrero de 2021/Aceptado: 30 de abril de 2021

\section{RESUMEN}

Objetivo: elaborar una aproximación a la prevalencia de los problemas de sueño (PDS) en mujeres climatéricas colombianas durante la pandemia COVID-19.

Materiales y métodos: estudio transversal que pertenece al proyecto de investigación Calidad de Vida en la Menopausia y Etnias Colombianas bajo condiciones de pandemia [CAVIMEC+COVID STUDY]. Se incluyeron mujeres naturales y residentes en Colombia entre 40 y 59 años, quienes en los primeros cinco días de junio del 2020 participaron de forma anónima y voluntaria, previo consentimiento informado en el diligenciamiento de un formulario alojado en una plataforma virtual. Los PDS fueron identificados con el tercer ítem de Menopause Rating Scale. Se exploraron características sociodemográficas, la presencia y severidad de los PDS y el estado menopáusico. Se hace estadística descriptiva.

\footnotetext{
* Correspondencia: Álvaro Monterrosa Castro. La Matuna. Avenida Venezuela. Edificio City Bank. Oficina 6-A. Cartagena, Colombia.

1. Médico. Especialista en Ginecología y obstetricia. Líder del Grupo de Investigación Salud de la Mujer. Profesor titular. Facultad de Medicina. Universidad de Cartagena, Cartagena (Colombia).

2. Médico. Integrante Grupo de Investigación Salud de la Mujer. Candidata a Magister en Epidemiologia Clínica. Fundación Universitaria Ciencias de la Salud (FUCS), Bogotá (Colombia).
}

Resultados: participaron 984 mujeres, la mediana de edad fue 47,0 [RIC: 42,0-53,5] años. El $84,5 \%$ de las participantes eran mestizas, el 13,7\% afrodescendientes y 1,7\% indígenas. El 39,3\% posmenopáusicas. El 70\% residían en la región caribe colombiana. Informaron PDS 637 (64,7\%) de las participantes y 112 (11,3\%) tenían PDS severos. Las posmenopáusicas informaron un 65,1\% de PDS, en forma severa el 10,1\%, y las premenopáusicas informaron 64,5\%, en forma severa el 12,2\%.

Conclusión: los PDS podrían ser un problema frecuente en las mujeres en estado premenopáusico y postmenopáusico, en este periodo de pandemia. Se debe explorar este problema en la consulta ginecológica para ofrecer soluciones. Se requieren estudios poblacionales que confirmen estas observaciones.

Palabras clave: trastornos del sueño del ritmo circadiano, pandemias, menopausia, climaterio, coronavirus, persona de mediana edad.

\section{ABSTRACT}

Objective: To make an aproximation to the prevalence of sleep disorders in Colombian menopausal women during the COVID-19 pandemic.

Material and methods: Cross-sectional study as part of the Quality of Life in Menopause and Colombian 
Ethnic Groups research project [CAVIMEC+COVID STUDY]. The population consisted of women born and residing in Colombia, 40 to 59 years of age, who signed an informed consent and agreed to participate by completing an online form, freely and anonymously, in the first five days of June 2020. Sleep disorders were identified using the third item on the Menopause Rating Scale. Sociodemographic characteristics, presence and severity of sleep disorders and menopause status were explored. Descriptive statistics are provided.

Results: Overall, 984 women aged 47.0 [IQR: 42.053.5] years were included: $84.5 \%$ mestizo, $13.7 \%$ Afro-Colombian, 1.7\% indigenous; $39.3 \%$ were postmenopausal; 70\% lived in the Caribbean region of Colombia. Sleep disorders were reported by 637 women (64.7\%), and 112 (11.3\%) had severe sleep disorders. Among postmenopausal women, 65.1\% reported sleep disorders with $10.1 \%$ reporting severe disorders, while $64.5 \%$ of premenopausal reported sleep disorders, and 12.2\% severe disorders.

Conclusion: Sleep disorders could be a frequent problem among premenopausal as well as postmenopausal women in the pandemic time. This issue should be explored during gynecological visits in order to offer solutions. Population studies that confirm these observations are required.

Keywords: Circadian rhythm disorders, sleep disorders, pandemics, menopause, coronavirus, middle aged individual.

\section{INTRODUCCIÓN}

Las diferentes etapas del ciclo vital humano se acompañan de experiencias y transformaciones que se relacionan con el entorno en el que los individuos se desarrollan. Identificar las problemáticas que predominan en cada una de esas fases permitirá implementar intervenciones que redunden en la conservación de la buena calidad de vida $(1,2)$.

El climaterio es una de las etapas vitales de la mujer; tiene en su interior el último periodo menstrual o menopausia, que sucede cuando los ovarios disminuyen la producción de estrógenos y se suele acompañar de di- versos síntomas o percepciones sintomatológicas, varias de las cuales poseen importante relevancia clínica. La menopausia se reconoce luego de doce meses consecutivos sin menstruación, es un evento natural y marca el fin de la fertilidad. Los años previos a la menopausia se denominan premenopausia (pueden involucrar la transición a la menopausia) y los posteriores a un año de cese definitivo menstrual, posmenopausia $(3,4)$.

Simultáneamente con los cambios biológicos intervienen en la magnitud de la sintomatología relacionada con el climaterio el rol de la mujer (jubilación profesional, síndrome del nido vacío, personalidad y estado de ánimo, desempeño social, consideraciones motivacionales), las dinámicas socio familiares (la vejez, la muerte de los padres y las dificultades en la relación de pareja), así como el significado que tiene la menopausia en su entorno social, el posicionamiento femenino y las influencias culturales $(2,4,5)$.

Son varios los síntomas menopáusicos que se han señalado: oleadas de calor, taquicardia, sudoración, sequedad y disfunción urogenital o sexual, irritabilidad, ansiedad, angustia, labilidad emocional, dolores musculares y articulares, y problemas del sueño (PDS) (2-5). Sobre estos últimos, se han estudiado los despertares nocturnos, el insomnio, el alargamiento de la fase de latencia, la pérdida del mantenimiento de las fases del sueño, la somnolencia diurna, la reducción del estado de alerta y, por lo tanto, la eficacia y la calidad del sueño $(6,7)$. Los PDS se han asociado al estado de ánimo depresivo, ansiedad y estrés. Inducen disminución de la calidad de vida, están relacionados con problemas crónicos sanitarios, son marcadores de riesgo cardiovascular y, además, son considerados un problema de salud pública (7-12).

Numerosos factores biopsicosociales alteran la arquitectura del sueño. Uno de los acontecimientos que puede desencadenar o agudizar colectiva e individualmente los PDS, es una pandemia. Incertidumbre, miedo, estrés y ansiedad; se ha observado que han hecho presencia simultáneamente en la pandemia COVID-19 (8-10). A finales de enero del 2020, la Organización Mundial de la Salud (OMS) declaró al COVID-19 como una emergencia de salud pública 
de interés internacional; semanas más tarde fue categorizada como pandemia (13). A finales de junio del 2021 se señalaron a nivel mundial más de 182 millones de contagios y más de tres millones novecientos mil personas fallecidas (14).

La cuarentena fue una de las medidas sugeridas por organizaciones internacionales de salud, con el fin de hacerle frente a la pandemia COVID-19 $(10,15,16)$. Es definida como el distanciamiento temporal impuesto a una población, persona o grupo por razones de seguridad $(17,18)$. Tiene como ventaja reducir la propagación de la enfermedad; sin embargo, contribuye al desarrollo de situaciones adversas, especialmente despidos laborales, deterioro económico, necesidad de improvisar condiciones para el trabajo en casa, inestabilidad emocional, estrés postraumático, pobre concentración, indecisión, exige adecuación personal a una nueva forma de convivencia, ocasiona distanciamiento familiar y aumento en la percepción de soledad $(10,17,18)$. Varios autores $(15,19,20)$ han indicado correlación directa entre aislamiento social, percepción de soledad, calidad de vida en la menopausia y la calidad del sueño. Así mismo, un metaanálisis indicó relación entre la falta de apoyo social y los PDS (21).

En Colombia, para junio de 2020, el gobierno nacional decretó una cuarentena obligatoria y toque de queda en las grandes ciudades a consecuencia del COVID-19. Los contagios y muertes se incrementaban a diario, sin desbordar la capacidad de atención sanitaria. De esta manera, se consideró importante evaluar el efecto de estas medidas, en la salud mental y la calidad de vida en la población en general y en ciertos grupos poblacionales, entre ellos niños, adolescentes y mujeres en etapa del climaterio. Aún son insuficientes los estudios que evalúan la percepción sintomatológica de los PDS en población climatérica latinoamericana bajo el contexto de una epidemia o pandemia. El objetivo de este estudio fue elaborar una aproximación a la prevalencia de los PDS en un grupo de mujeres colombianas en etapa de climaterio durante la cuarentena obligatoria instaurada gubernamentalmente a consecuencia de la pandemia del COVID-19.

\section{MATERIALES Y MÉTODOS}

Diseño y población: estudio de corte transversal. Participaron mujeres cuyas edades se encontraban entre 40 y 59 años, de nacionalidad colombiana y residentes en Colombia, ninguna estaba embarazada, sabían leer, escribir, tenían acceso y habilidades mínimas en el manejo de redes sociales o plataformas digitales, se declararon auto saludables por el hecho de realizar las labores cotidianas, carecer de discapacidad física o mental y sentirse bien según su percepción. Se excluyeron aquellas mujeres que no comprendieron las herramientas de estudio o que diligenciaron el formulario de manera incompleta. Se hizo muestreo consecutivo de las mujeres que participaron voluntariamente en el estudio. La muestra estuvo constituida por las mujeres que contestaron la encuesta entre el 1 y el 5 de junio de 2020.

Procedimiento: se hizo una convocatoria abierta para participar en el estudio por medio de redes sociales (Facebook, Instagram y WhatsApp) como parte del proyecto de investigación CAVIMEC+COVID STUDY (Calidad de Vida en la Menopausia y Etnias Colombianas bajo condiciones de pandemia). Otros informes finales derivados del mismo proyecto han sido publicados recientemente $(19,20)$. A las mujeres interesadas en ingresar al estudio se les informó el carácter anónimo y voluntario de este; no recibieron incentivos de ningún tipo por participar. Se les invitó a ingresar al siguiente link: https://docs.google.com/ forms/d/e/1FAIpQLSeh_as86kzZp6Tu1K2gFgZlpITumfO7j8jDGyop9GkWiVNbMg/viewform?v $\mathrm{c}=0 \& \mathrm{c}=0 \& \mathrm{w}=1 \&$ gxids $=7628$. El propósito era diligenciar el formulario electrónico especialmente diseñado, donde se trascribió la información referente a las características sociodemográficas, su estado en relación con la menopausia y el tercer ítem de la escala Menopause Rating Scale (MRS), que identifica el síntoma PDS al interrogar sobre la dificultad para conciliar el sueño, dificultad para dormir o despertar más temprano de lo deseado. La MRS hace una exploración de diversos síntomas relacionados con la menopausia por medio de once preguntas, ha sido traducida y validada en varios idiomas, con aplicación 
en varios grupos de mujeres colombianas (20-22). El tercer ítem se responde tipo Likert: ausencia de PDS, existencia leve, moderada, severa o muy severa, asignándose una calificación que va desde cero hasta cuatro puntos, respectivamente (4). A su vez, en el presente proyecto se hizo la valoración conjunta de PDS severos y muy severos (puntajes de 3 y 4) y fueron redefinidos como PDS intensos (19). Los datos fueron depurados, los correos electrónicos de las participantes se eliminaron para preservar el anonimato y las formas incompletas fueron descartadas.

Variables medidas: edad, aspectos referentes a los episodios menstruales y número de hijos, región colombiana donde residía, etnicidad (mestiza, afrodescendiente e indígena), la cual fue definida por auto reconocimiento; estado menopáusico: premenopausia (menstruación regular, irregular o amenorrea menor a un año) y posmenopausia (amenorrea superior a un año). Se midió la frecuencia y severidad de los PDS.

Análisis: el análisis estadístico fue realizado en Stata/IC 16.0. Los datos continuos son expresados en mediana y rango intercuartílico [RIC] o en medias y desviación estándar, de acuerdo con la distribución de los datos. Los datos categóricos se presentan en valores absolutos y porcentuales. Se estableció la frecuencia de PDS para las climatéricas (todas las participantes), las premenopáusicas y las posmenopáusicas, y según las regiones geográficas colombianas donde residían las participantes.

Declaración sobre aspectos éticos: el proyecto CAVIMEC-COVID19 tiene en cuenta las recomendaciones de la Declaración de Helsinki y el informe Belmont. También la resolución 8430 de la República de Colombia, que establece pautas científicas y administrativas para la investigación en humanos. Está clasificado como un estudio de riesgo mínimo y posee aval institucional de la Universidad de Cartagena, Colombia, y fue aprobado por el Comité de Ética de la Clínica Santa Cruz de Bocagrande, Cartagena, Colombia, acta 03-2020 del 21 de marzo 2020.

\section{RESULTADOS}

En los primeros cinco días de junio de 2020 se recibieron 1,012 formularios, 28 (2,7\%) tuvieron datos incompletos y fueron descartados. Se incluyó en el análisis un total de 984 mujeres, la mediana de edad de la muestra fue 47,0 años [RIC: 42,0-53,5]. Del total, el 84,5\% de las mujeres encuestadas eran mestizas, el 13,7\% afrodescendientes y el 1,7\% indígenas; 39,3\% estaban en posmenopausia. La mediana del número de hijos fue: Me [RIC] 2,0 [1,0-2,0] (Tabla 1).

\begin{tabular}{|c|c|c|c|}
\hline \multicolumn{4}{|c|}{$\begin{array}{l}\text { Tabla } 1 . \\
\text { Características sociodemográficas de las mujeres encuestadas en época de pandemia } \\
\text { por Covid } 19 \text { en Colombia, } 2020 .\end{array}$} \\
\hline & $\begin{array}{c}\text { Climatéricas } \\
\mathbf{n}=984 \\
\end{array}$ & $\begin{array}{c}\text { Premenopáusicas } \\
n=598(60,7 \%)\end{array}$ & $\begin{array}{c}\text { Posmenopáusicas } \\
n=386(39,3 \%)\end{array}$ \\
\hline Edad, Me [RIC] & $47,0[42,0-53,5]$ & $43,0[40,0-46,0]$ & $54,0[52,0-57,0]$ \\
\hline Hijos, Me [RIC] & $2,0[1,0-2,0]$ & $2,0[1,0-2,0]$ & $2,0[1,0-3,0]$ \\
\hline 40-44 años, n (\%) [IC95] & $392(39,8)$ & $384(64,2)$ & $8(2,0)$ \\
\hline 45-49 años, n (\%) [IC95] & $189(19,2)$ & $148(24,7)$ & $41(10,6)$ \\
\hline 50-54 años, n (\%) [IC95] & $211(21,5)$ & $58(9,7)$ & $153(39,6)$ \\
\hline 55-59 años, n (\%) [IC95] & $192(19,5)$ & $8(1,3)$ & $184(47,6)$ \\
\hline Mestizas, n (\%) [IC95\%] & $832(84,6)$ & $493(82,4)$ & $339(87,8)$ \\
\hline Afrodescendientes, n (\%) [IC95\%] & $135(13,7)$ & $91(15,2)$ & $44(11,4)$ \\
\hline Indígenas, n (\%) [IC95\%] & $17(1,7)$ & $14(2,4)$ & $3(0,8)$ \\
\hline
\end{tabular}


El 79.9 \% de la población encuestada vivía en la región de la Costa Caribe colombiana, mientras que en la región Andina vivía el 17,1\% de las mujeres incluidas en el estudio, y el 3\% pertenecía a otras regiones (Amazónica, Pacífica, Orinoquia e Insular).

Se identificaron 386 mujeres posmenopáusicas (39\%). En este subgrupo se encontró que la mediana de la edad en que se presentó la última menstruación fue 49,0 [RIC: 47,0-51,0] años. A su vez, la mediana del número de años de posmenopausia que tenía este subgrupo de mujeres fue de 5,0 [RI: 3,0-7,0] años. 229 (23,2\%) estaban en postmenopausia temprana [1-5 años], $133(13,5 \%)$ en posmenopausia tardía (6-10 años), mientras que 34 (2,4\%) tenían once o más años de estar con cese definitivo menstrual. Las características sociodemográficas del subgrupo posmenopáusicas y premenopáusicas se presentan en la Tabla 1.

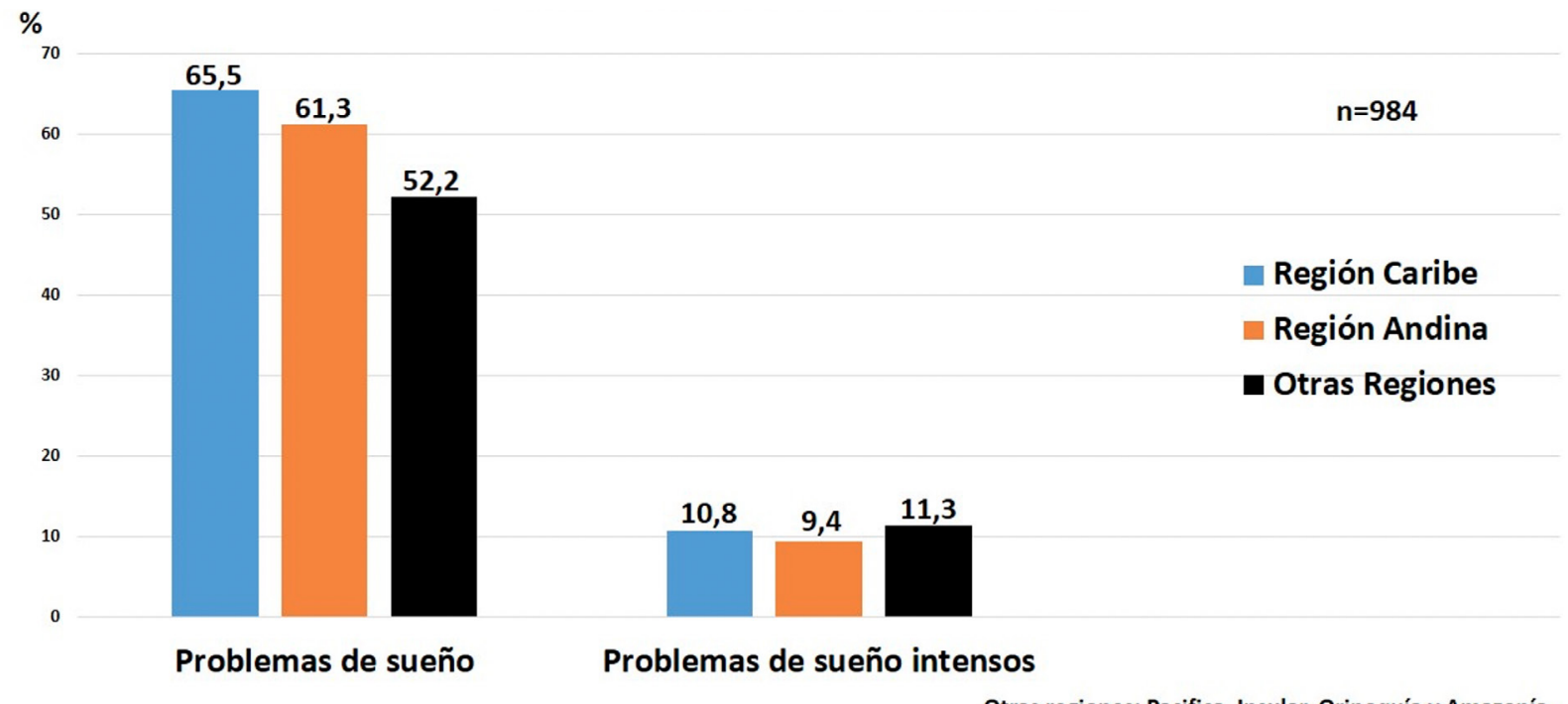

Figura 1. Frecuancia porcentual de problemas de sueño y problemas de sueño intenso según regiones geográficas colombianas.

De las participantes, 637 (64,7\%) manifestaron PDS; 112 (11,3\%) del total de mujeres manifestaron tener PDS intensos. La figura 1 presenta las frecuencias de los PDS y PDS intensos, según las regiones geográficas colombianas donde residían las participantes. Las mujeres posmenopáusicas in- formaron ligeramente mayor presencia de PDS que las premenopáusicas, $65,1 \%$ frente a $64,5 \%$, respectivamente. Respecto a los PDS intensos, afectaron al 10,0\% de las posmenopáusicas y al 12,2\% de las premenopáusicas (Tabla 2).

\begin{tabular}{|c|c|c|c|}
\hline & $\begin{array}{c}\text { Climatéricas } \\
\mathbf{n}=984\end{array}$ & $\begin{array}{c}\text { Premenopáusicas } \\
n=598\end{array}$ & $\begin{array}{c}\text { Posmenopáusicas } \\
n=386\end{array}$ \\
\hline Presentes & $637(64,7)$ & $386(64,5)$ & $251(65,0)$ \\
\hline Leves & $316(32,2)$ & $189(31,6)$ & $127(32,9)$ \\
\hline Moderados & $209(21,2)$ & $124(20,7)$ & $85(22,0)$ \\
\hline Severos & $76(7,8)$ & $52(8,7)$ & $24(6,2)$ \\
\hline Muy severos & $36(3,6)$ & $21(3,5)$ & $15(3,8)$ \\
\hline
\end{tabular}




\section{DISCUSIÓN}

En el estudio se observó que en un poco más del 64\% de las mujeres climatéricas incluidas hay presencia de PDS, explorado como un síntoma menopaúsico. La cifra fue similar cuando las participantes se exploraron agrupadas según las regiones geográficas colombianas donde residían o según el estado menopaúsico en el cual se encontraban.

La mala calidad del sueño suele ser un síntoma común entre los adultos, usualmente superior a la indicada por los más jóvenes. Se ha señalado que el 50\% de las personas de más de cincuenta años experimentan PDS (25). Monterrosa-Castro et al. (22), utilizando MRS en 1215 mujeres climatéricas del caribe colombiano, con edad promedio de 49 años, una década antes de la pandemia COVID-19, encontraron PDS en el 47,2\% de las estudiadas. A su vez, notificaron que los PDS leves fueron del 16,7\%, y los muy severos del 1,2\%; es decir, menor a lo encontrado en estas mujeres evaluadas bajo condición de cuarentena por la pandemia del COVID-19. Un estudio en población norteamericana indicó que el crecimiento en el número de casos por COVID-19 se acompañó de incremento en los niveles de los PDS, ansiedad, estrés y depresión (26). Silva et al. (8), señalaron que la actual pandemia no solo alteró la rutina de una gran proporción de la población, también afectó su calidad de sueño. Similar a los datos que hemos encontrado, un estudio llevado a cabo con población italiana durante la misma pandemia por COVID-19 indicó presencia de disturbio del sueño en el 54\% de la población, e indicaron que el insomnio, la pérdida y la mala calidad del sueño eran manifestaciones generalizadas con tasas similares a las encontradas en otras grandes crisis, por ejemplo, desastres naturales como terremotos, inundaciones o incendios forestales (9).

El estudio SWAN (Study of Women's Health Across the Nation) ha señalado que, en general, la prevalencia de los PDS cambia entre el 16\% y el $42 \%$ en la premenopausia, en la perimenopausia entre el 39\% y el $47 \%$, y en la posmenopausia entre el 35\% y el $60 \%$ (27). Por tanto, se observan diferencias en las frecuencias de los PDS entre estados menopaúsicos, con tendencia a ser más frecuentes en la posmenopausia. Los hallazgos de nuestra evaluación son coherentes con dichos señalamientos. Además, en el grupo que hemos evaluado, los PDS intensos fueron más frecuentes entre las que aún menstruaban que entre las posmenopáusicas. Son pocos los estudios que valoran los PDS, específicamente en mujeres en etapa climatérica dentro de la actual pandemia por el COVID-19 $(19,20)$.

Nuestros datos son superiores a los informados por Pappa et al. (28), en un metaanálisis de cinco estudios identificaron prevalencia de insomnio del $34,3 \%$ en trabajadores del área de la salud. Por su parte, Kokou-Kpolou et al. (29), en un estudio que contó con población general francesa bajo confinamiento por la pandemia COVID-19, que incluía un 75,5\% de mujeres, observaron insomnio en el 19.1\%. Además, Gualano et al. (10), quienes trabajaron con población general italiana bajo confinamiento por COVID-19, encontraron a través de un cuestionario de calidad de la salud, que el 42,2\% manifestaron PDS, y entre ellos, en el 17,4\% observó insomnio moderado y severo. En población colombiana, recientemente se han publicado estudios de Monterrosa-Castro et al. (30) y de Parra-Saavedra et al. (31). Los primeros en 531 médicos generales colombianos, 59,5\% mujeres, evaluados durante la pandemia COVID-19 para establecer estrés laboral, miedo y síntomas psicosomáticos relacionados con la pandemia, encontraron que el 32,7\% presentaban pesadillas con el virus, el 43,1\% se sentían imposibilitados para dormir, y el 53,9\% no dormían porque les preocupaba el COVID-19. En el otro estudio, llevado a cabo con 946 gestantes colombianas, el 49,1\% manifestaron presentar insomnio, al interrogarles diferentes síntomas psicológicos. Las diferencias entre los estudios se explican por las herramientas de medición utilizadas, como las situaciones medio ambientales en las cuales se encontraban las evaluadas.

El estudio tiene como fortaleza ser de los primeros estudios latinoamericanos en explorar uno 
de los síntomas menopaúsicos, específicamente los PDS, durante la pandemia COVID-19. La invitación y toma de la información por vía electrónica es, al tiempo, fortaleza y limitación. Permitió obtener datos de forma rápida, con bajo costo y llegar a regiones geográficas distantes. No obstante, no se puede comprobar el cumplimiento de los criterios de inclusión. Es difícil poder controlar sesgos de selección, por ejemplo, solo se puede involucrar población con equipos de suficiente gama, acceso a redes virtuales y destrezas en el manejo de recursos digitales. Posee como limitaciones posible sesgo de medición, utilizar el ítem de una escala de calidad de vida, aunque sea de uso universal y específicamente para mujeres en climaterio. A su vez, no haber interrogado sobre situaciones o hábitos que afectan la calidad del sueño, uso de medicamentos o infusiones para dormir, diagnóstico previo de patologías del sueño e intervenciones y comorbilidades físicas o mentales. Tiene las limitaciones de los diseños transversales y su alcance fue descriptivo de los síntomas subjetivos de PDS, ya que no es posible, en exploraciones comunitarias, utilizar métodos objetivos como la polisomnografía. Se ameritan estudios en mujeres en climaterio, donde se aborden las patologías del dormir, durante situaciones críticas como una pandemia.

$\mathrm{Al}$ proteger el sueño, durante la pandemia y otros eventos catastróficos, se fomentan la resiliencia y el afrontamiento para hacer frente al encierro social, la atípica convivencia, la angustia, la precepción de soledad y la incertidumbre $(19,20,32)$. El sueño es un proceso vital que mantiene la homeostasis y la calidad de vida, se relaciona con sensación de bienestar y buena salud mental. El sueño es uno de los tres pilares de la salud sostenible, los otros dos son la alimentación y el ejercicio físico $(8,32)$.

\section{CONCLUSIÓN}

En un grupo de mujeres colombianas de entre 40 y 59 años, que fueron abordadas por medio de redes sociales se encontró que el 64,7\% informaron tener la percepción de experimentar PDS. A su vez, cerca del 10\% de todas las estudiadas manifestaron que los PDS eran intensos. Los ginecólogos y los demás profesionales de la salud que atienden mujeres en climaterio deben interrogar sistemáticamente sobre síntomas que indiquen alteración en la calidad del sueño. Se deben hacer estudios de base poblacional que evalúen la magnitud de este problema y otras alteraciones de la calidad de vida relacionadas.

\section{FUENTES DE FINANCIACIÓN}

La Universidad de Cartagena, por medio del Plan de Fortalecimiento para Grupos de Investigación categorizados por MIN-CIENCIAS, según resolución 01430-2019 y acta 064-2019, apoyó logísticamente la realización del presente estudio. Los autores no recibieron honorarios por el desarrollo de la investigación. La universidad de Cartagena no participó en el diseño del estudio, recopilación, análisis, interpretación de los datos, redacción del informe ni en la decisión de someter los resultados a publicación.

\section{REFERENCIAS}

1. Strauss JFB \& Robert L. Yen \& Jaffe's Reproductive Endocrinology: Physiology, Pathophysiology, and Clinical Management, Seventh Edition. (Saunders, 2014). https://www.elsevier.com/books/yen-and-jaffes-reproductive-endocrinology/strauss/978-1-4557-2758-2

2. Takeda Y. Understanding the Life Stages of Women to Enhance Your Practice. JMAJ. 2010;53(5): 273-278. https:// www.med.or.jp/english/journal/pdf/2010_05/273_278.pdf

3. Blümel JE, Lavín P, Vallejo MS, Sarrá S. Menopause or climacteric, just a semantic discussion or has it clinical implications? Climacteric. 2014;17(3):235-41. https://doi.org/10.3109/13697137.2013.838948

4. Bacon JL. The Menopausal Transition. Obstet Gynecol Clin North Am. 2017; 44(2):285-296. https://doi. org/10.1016/j.ogc.2017.02.008

5. Quiroga A, Larroy C, González-Castro P. Climacteric symptoms and their relation to feminine self-concept. Climacteric. 2017;20(3):274-279. https://doi.org/10 $.1080 / 13697137.2017 .1310192$ 
6. Gooneratne NS, Vitiello MV. Sleep in older adults: normative changes, sleep disorders, and treatment options. Clin Geriatr Med. 2014; 30(3):591-627. https://doi.org/10.1016/j.cger.2014.04.007

7. Baker FC, de Zambotti M, Colrain IM, Bei B. Sleep problems during the menopausal transition: prevalence, impact, and management challenges. Nat Sci Sleep. 2018; 10:73-95. https://doi.org/10.2147/NSS. S125807

8. Silva ESME, Ono BHVS, Souza JC. Sleep and immunity in times of COVID-19. Rev Assoc Med Bras. 2020; 66(Suppl 2):143-147. http://dx.doi. org/10.1590/1806-9282.66.S2.143

9. Cellini N, Canale N, Mioni G, Costa S. Changes in sleep pattern, sense of time and digital media use during COVID-19 lockdown in Italy. J Sleep Res. 2020; 29(4): e13074. http://dx.doi.org/10.1111/jsr.13074

10. Gualano MR, Lo Moro G, Voglino G, Bert F, Siliquini R. Effects of Covid-19 Lockdown on Mental Health and Sleep Disturbances in Italy. Int J Environ Res Public Health. 2020; 17(13):4779. http://dx.doi. org/10.3390/ijerph17134779.

11. Huang Y, Zhao N. Generalized anxiety disorder, depressive symptoms and sleep quality during COVID-19 outbreak in China: a web-based cross-sectional survey. Psychiatry Res. 2020; 288:112954. http:// dx.doi.org/10.1016/j.psychres.2020.112954

12. Xu Q, Lang CP. Examining the relationship between subjective sleep disturbance and menopause: a systematic review and meta-analysis. Menopause. 2014; 21(12):1301-18. https://doi.org/10.1097/ GME.0000000000000240

13. PAHO/WHO. WHO characterizes COVID-19 as a pandemic [Internet]. Ginebra. [consultado 2021 febrero 8]. Disponible en: https://www.paho.org/hq/index. php?option $=$ com_content\&view $=$ article\&id $=15756: \mathrm{w}$ ho-characterizes-covid-19-as-a-pandemic\&Itemid = 192 6\&lang $=\mathrm{en}$

14. Johns Hopkins University (JHU). COVID-19 Dashboard by the Center for Systems Science and Engineering (CSSE) at Johns Hopkins University (JHU). [Internet]. [Consultado: 2021]. Disponible: https:// coronavirus.jhu.edu/map.html
15. Xiao H, Zhang Y, Kong D, Li S, Yang N. Social Capital and Sleep Quality in Individuals Who Self-Isolated for 14 Days During the Coronavirus Disease 2019 (COVID-19) Outbreak in January 2020 in China. Med Sci Monit. 2020; 26: e923921. http://dx.doi. org/10.12659/MSM.923921

16. Brooks SK, Webster RK, Smith LE, Woodland L, Wessely S, Greenberg N, et al. The psychological impact of quarantine and how to reduce it: rapid review of the evidence. Lancet. 2020; 395(10227):912-920. https://doi.org/10.1016/S0140-6736(20)30460-8

17. Yu B, Steptoe A, Niu K, Ku PW, Chen LJ. Prospective associations of social isolation and loneliness with poor sleep quality in older adults. Qual Life Res. 2018; 27(3):683691. https://doi.org/10.1007/s11136-017-1752-9

18. Cho JH, Olmstead R, Choi H, Carrillo C, Seeman TE, Irwin MR. Associations of objective versus subjective social isolation with sleep disturbance, depression, and fatigue in community-dwelling older adults. Aging Ment Health. 2019; 23(9):1130-1138. https://doi.or g/10.1080/13607863.2018.1481928

19. Monterrosa-Castro Á, Monterrosa-Blanco A, GonzálezSequeda A. Perceived Loneliness and Severe Sleep Disorders in Adult Women during the Covid-19 Quarantine: A Cross-Sectional Study in Colombia. J Prim Care Community Health. 2021; 12:21501327211025170. https:// doi.org/10.1177/21501327211025170

20. Monterrosa-Blanco A, Monterrosa-Castro A, GonzalezSequeda A. Online assessment of the perception of loneliness and associated factors in Colombian climacteric women during the COVID-19 pandemic: A cross-sectional study. Health Promotion Perspectives, 2021;11(2):230-239. https://doi. org/10.34172/hpp.2021.28

21. Uchino BN, Trettevik R, Kent de Grey RG, Cronan S, Hogan J, Baucom BRW. Social support, social integration, and inflammatory cytokines: A meta-analysis. Health Psychol. 2018; 37(5):462-471. http://dx.doi. org/10.1037/hea0000594

22. Monterrosa-Castro A, Romero-Pérez l, PaterninaCaicedo Á. Manifestaciones músculo-articulares, y no las oleadas de calor, es el síntoma más prevalente en mujeres climatéricas del Caribe colombiano. Salud Uninorte, Barranquilla. 2010; 26(2):179-188. http:// www.scielo.org.co/pdf/sun/v26n2/v26n2a02.pdf 
23. Monterrosa-Castro A, Paternina-Caicedo A, RomeroPérez Ivette. La calidad de vida de las mujeres en edad media varía según el estado menopáusico. Iatreia. 2011; 24(2):136-145. https://revistas.udea.edu.co/ index.php/iatreia/article/view/9599/8843

24. Heinemann K, Ruebig A, Potthoff P, Schneider HP, Strelow F, Heinemann LA, et al. The Menopause Rating Scale (MRS) scale: a methodological review. Health Qual Life Outcomes. 2004; 2(2):45. https:// doi.org/10.1186/1477-7525-2-45

25. McHugh JE, Lawlor BA. Perceived stress mediates the relationship between emotional loneliness and sleep quality over time in older adults. Br J Health Psychol. 2013; 18(3):546-55. https://doi.org/10.1111/j.20448287.2012.02101.x

26. Voitsidis P, Gliatas I, Bairachtari V, Papadopoulou K, Papageorgiou G, Parlapani E, et al. Insomnia during the COVID-19 pandemic in a Greek population. Psychiatry Res. 2020; 289:113076. http://dx.doi. org/10.1016/j.psychres.2020.113076

27. Lee J, Han Y, Cho HH, Kim MR. Sleep Disorders and Menopause. J Menopausal Med. 2019; 25(2):83-87. http://dx.doi.org/10.6118/jmm. 19192

28. Pappa S, Ntella V, Giannakas T, Giannakoulis VG, Papoutsi E, Katsaounou P. Prevalence of depression, anxiety, and insomnia among healthcare workers during the COVID-19 pandemic: A systematic review and meta-analysis. Brain Behav Immun. 2020; 88:901907. http://dx.doi.org/10.1016/j.bbi.2020.05.026

29. Kokou-Kpolou CK, Megalakaki O, Laimou D, Kousouri M. Insomnia during COVID-19 pandemic and lockdown: Prevalence, severity, and associated risk factors in French population. Psychiatry Res.
2020; 290:113128. http://dx.doi.org/10.1016/j.psychres. 2020.113128

30. Monterrosa-Castro A, González-Sequeda A, BeltránBarrios T. Percepción de discriminación en un grupo de médicos generales colombianos durante la pandemia del COVID-19 y su relación con factores laborales y psicológicos. Salud Uninorte, Barranquilla. 2020; 36(1):25-45. Available from: http://www.scielo. org.co/scielo.php?script $=$ sci_arttext\&pid $=$ S0120 $55522020000100025 \& \operatorname{lng}=\mathrm{en}$

31. Parra-Saavedra M, Villa-Villa I, Pérez-Olivo J, GuzmanPolania L, Galvis-Centurion P, Cumplido-Romero Á, et al. Attitudes and collateral psychological effects of COVID-19 in pregnant women in Colombia. Int J Gynaecol Obstet. 2020;151(2):203-208. http:// dx.doi.org/10.1002/ijgo.13348

32. Morin CM, Carrier J, Bastien C, Godbout R; Canadian Sleep and Circadian Network. Sleep and circadian rhythm in response to the COVID-19 pandemic. Can J Public Health. 2020; 111(5):654-657. http://dx.doi. org/10.17269/s41997-020-00382-7

\section{CONTRIBUCIÓN DE LOS AUTORES}

Álvaro Monterrosa-Castro: diseño del estudio, revisión del análisis estadístico, supervisión de la escritura, revisión final y aprobación definitiva del documento.

Angélica Monterrosa-Blanco: análisis estadístico, escritura, revisión final y aprobación definitiva del documento. 